\title{
Asymmetric energy flow in coupled nonlinear $L C$ transmission line
}

\author{
XU Wen ${ }^{1}$, CHEN WeiZhong ${ }^{1 *}$, TAO Feng ${ }^{1,2} \&$ PAN JunTing ${ }^{1}$ \\ ${ }^{1}$ Key Laboratory of Modern Acoustics, Ministry of Education, and Institution of Acoustics, Nanjing University, Nanjing 210093, China; \\ ${ }^{2}$ School of Electrical Engineering \& Information, Anhui University of Technology, Ma'anshan 243002, China
}

Received March 2, 2011; accepted April 19, 2011

\begin{abstract}
We constructed a coupled $L C$ transmission line and studied the propagation of waves in it. We found asymmetric energy flow when we changed the driving conditions at the boundary. We analyzed this change and believe that it occurs because of the bandpass characteristics of the $L C$ transmission line and high-order harmonic waves induced by nonlinearities. The $L C$ transmission line could be used to simulate a microscopic crystal lattice. Therefore, we hope to observe thermal rectification in the system. We investigated the dependence of the system on different parameters, and then discussed the multi-frequency condition to aid in experimental verification.
\end{abstract}

nonlinearity, $L C$ transmission line, asymmetric energy flow, passband, fundamental wave, harmonic wave

Citation: $\quad \mathrm{Xu} \mathrm{W}$, Chen W Z, Tao F, et al. Asymmetric energy flow in coupled nonlinear LC transmission line. Chinese Sci Bull, 2011, 56: 3087-3091, doi: 10.1007/s11434-011-4665-y

Recently, thermal rectification in one-dimensional (1D) systems has attracted a great deal of research interest [1-3]. For numerical simulations of a coupled 1D lattice, it has been found that energy can flow through the channel from the high-temperature thermal bath to the lower-temperature bath in one direction. However, the lattice behaves as an insulator if the thermal baths are swapped. An experiment using a nanotube has shown this behavior with a $7 \%$ difference between heat fluxes in the two opposite directions [3]. However, nanotubes are not strictly 1D lattices in reality. In fact, we should be able to observe this abnormal phenomenon in experiments based on a macroscopic 1D lattice, which has the similar governing equations as the microscopic crystal lattice. In a macroscopic system, the energy of vibration (phonon) of each lattice can be measured directly. There are many types of macroscopic 1D lattice models, for example, pendulum chains [4] and nonlinear $L C$ transmission lines [5]. While taking into account effect of thermal baths, we used the $L C$ transmission line as our investigation model. The realization of random vibrations is much simpler to achieve with an electrical signal than in a

*Corresponding author (email: wzchen@nju.edu.cn) mechanical system. In this work, we present a study of the propagation of waves in two coupled $L C$ transmission lines, in which one line exhibits nonlinear transmission [5] and the other line is linear. First, we investigated monofrequency driving through numerical simulation and looked for possible abnormal energy transmission. Then we further discus multi-frequency driving (thermal bath) to aid ensuring experimental realization.

\section{Coupled $L C$ transmission line}

The coupled $L C$ transmission line that we studied contains two sub-lines (Lines a and b), which have the same structure but different parameters. They are coupled by an inductor, $L_{\text {int }}$. This is shown in Figure 1. Each cell of the transmission lines has the usual form [6], which consists of one capacitor and two inductors. In Line a, the capacitor is nonlinear. A variable capacitance diode, BB112, working at a DC voltage near $V_{\mathrm{d}}=2.0 \mathrm{~V}$, serves as the nonlinear capacitor. If $\left|V_{n}\right|<2.0 \mathrm{~V}$, the capacitance is related to the AC voltage $V_{n}$ as follows [6]:

$$
C_{\mathrm{a}}\left(V_{n}\right)=C_{0}\left(1-2 \alpha V_{n}+3 \beta V_{n}^{2}\right),
$$




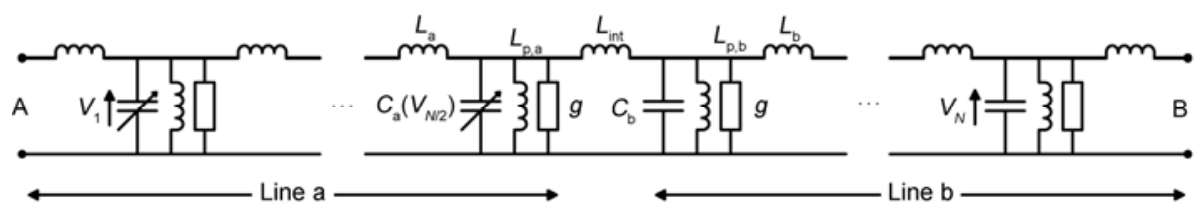

Figure 1 Coupled $L C$ transmission lines.

where the parameters are $C_{0}=320 \mathrm{pF}, \alpha=0.21 \mathrm{~V}^{-1}$ and $\beta=0.0197 \mathrm{~V}^{-2}$. In Line $\mathrm{b}$, the capacitor is linear, and $C_{\mathrm{b}}=C_{0}=320 \mathrm{pF}$. The series inductors $\left(L_{\mathrm{a}}, L_{\mathrm{b}}\right.$ and $\left.L_{\text {int }}\right)$ and the parallel inductors $\left(L_{\mathrm{p}, \mathrm{a}}\right.$ and $\left.L_{\mathrm{p}, \mathrm{b}}\right)$ are linear. The dissipation of these elements is taken into account. However, we only consider the dissipation contributions from the capacitor and the parallel inductor (main factor [7]), which are represented by the conductivity $(g)$. Furthermore, we assume that the dissipation is the same in the two sub-lines. In each line, according to Kirchhoff's law, we have

$$
\begin{aligned}
\frac{\mathrm{d}}{\mathrm{d} t}\left[C_{i}\left(V_{n}\right) \frac{\mathrm{d} V_{n}}{\mathrm{~d} t}\right] & =\frac{1}{L_{i}}\left(V_{n-1}+V_{n+1}-2 V_{n}\right) \\
& -\frac{1}{L_{\mathrm{p}, i}} V_{n}-g \frac{\mathrm{d} V_{n}}{\mathrm{~d} t},(i=\mathrm{a}, \mathrm{b}) .
\end{aligned}
$$

If the voltage is small $\left(V_{n}<<1\right)$ and the dissipation is weak, we can neglect the high order terms and dissipation terms in the equation. Consequently, we get the linearized equation:

$$
\begin{aligned}
\frac{\mathrm{d}^{2}}{\mathrm{~d} t^{2}} V_{n}= & \frac{1}{L_{i} C_{0}}\left(V_{n-1}+V_{n+1}-2 V_{n}\right) \\
& -\frac{1}{L_{\mathrm{p}, i} C_{0}} V_{n},(i=\mathrm{a}, \mathrm{b}) .
\end{aligned}
$$

In the uncoupled infinite-line condition, we can get the linear dispersion relation solution for plain waves:

$$
\omega_{i}^{2}=\omega_{0, i}^{2}+4 u_{0, i}^{2} \sin ^{2} \frac{k}{2},(i=\mathrm{a}, \mathrm{b}),
$$

where $\omega_{0, i}=1 / \sqrt{L_{\mathrm{p}, i} C_{0}}, u_{0, i}=1 / \sqrt{L_{i} C_{0}}(i=\mathrm{a}, \mathrm{b})$.

According to eq. (4), we get a lower cut-off (angular) frequency of $\omega_{i, \min }=\omega_{0, i}$ and an upper cut-off frequency of $\omega_{i, \max }=\sqrt{\omega_{0, i}^{2}+4 u_{0, i}^{2}}$. In the linear approximation, waves at frequency $\omega$ can propagate in the $L C$ line if $\omega_{\min } \leqslant \omega \leqslant \omega_{\max }$; otherwise they decay exponentially. This leads to the bandpass characteristics of the line. Both the lower and upper cut-off frequencies can be adjusted by changing these elementary parameters.

To investigate asymmetric energy flow in the coupled transmission line, we designed two sub-lines, with different passbands $\left[\omega_{\mathrm{a}, \min }, \omega_{\mathrm{a}, \max }\right]$ and $\left[\omega_{\mathrm{b}, \min }, \omega_{\mathrm{b}, \max }\right]$. This is shown in Figure 2.

The two sub-lines are coupled using a large inductor, $L_{\text {int }}$ $\left(L_{\text {int }}>L_{\mathrm{a}}, L_{\mathrm{b}}\right)$. Because $L_{\text {int }}$ is large, the coupling is weak, and we expect that the two sub-lines will retain their original

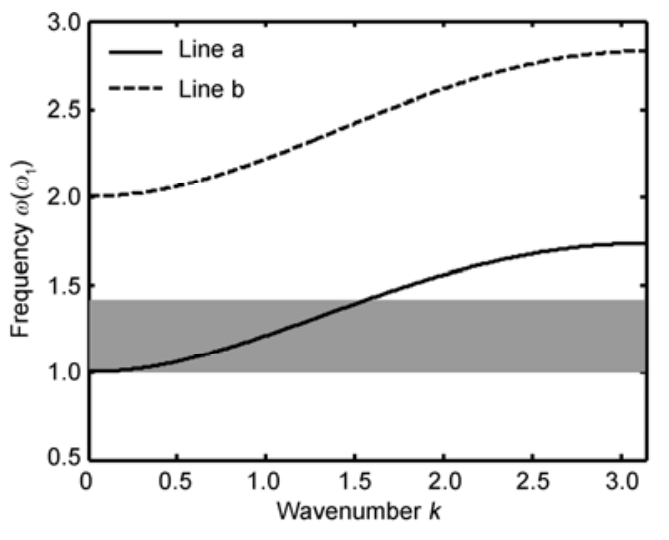

Figure 2 Dispersion curves for the uncoupled infinite lines, a and b. The asymmetric energy flow occurs at the frequencies in the shaded area.

characteristics, for example, their passbands. At the interface, the voltage satisfies

$$
L_{\text {int }} \frac{\mathrm{d} I_{\text {int }}}{\mathrm{d} t}=V_{N / 2}-V_{N / 2+1},
$$

where $I_{\text {int }}$ is the current passing through $L_{\text {int }}$.

A mono-frequency voltage, $V_{0} \sin \omega t$, serving as the driving frequency at one end the frequency, $\omega$, satisfies

$$
\omega_{\mathrm{a}, \min } \leqslant \omega \leqslant \omega_{\mathrm{a}, \max }
$$

and

$$
\omega<\omega_{\mathrm{b}, \min } \leqslant 2 \omega \leqslant \omega_{\mathrm{b}, \max } .
$$

Namely, $\omega$ is in the shaded area of Figure 2.

\section{Simulation}

The parameters of inductors are set to be $L_{\mathrm{p}, \mathrm{a}}=L_{0}=220 \mu \mathrm{H}$, $L_{\mathrm{p}, \mathrm{b}}=0.25 L_{0}, L_{\mathrm{a}}=2.0 L_{0}$ and $L_{\mathrm{b}}=1.0 L_{0}$. The capacitor parameters are the same as those in eq. (1). Therefore, Line a's passband is $\left[\omega_{1}, \sqrt{3} \omega_{1}\right]$, and Line b's passband is $\left[2 \omega_{1}, 2 \sqrt{2} \omega_{1}\right]$ with $\omega_{1}=1 / \sqrt{L_{a} C_{0}}$ (see Figure 2). The coupling inductance is $L_{\mathrm{int}}=4 L_{0}=880 \mu \mathrm{H}$, and the conductivity is $g=1.2 \times 10^{-5} \Omega^{-1}$. To approximate reasonable experimental conditions, we set the total number of cells to $N=40$, and each sub-line consists of 20 cells.

The driving frequency is $\omega=1.2 \omega_{1}$, which satisfies eqs. (6) and (7). To ensure the validity of eq. (1), we use a small amplitude $V_{0}=0.6 \mathrm{~V}$ to avoid unphysical results. The driving frequency is the input at one end of the transmission line, and the other end is connected to the ground. Therefore, if 
we drive at End A, the boundaries satisfy (Ends A, B correspond to $n=0, N+1$ )

$$
\begin{gathered}
V_{0}(t)=V_{0} \sin \omega t, \\
V_{N+1}(t)=0 .
\end{gathered}
$$

If we drive at End $\mathrm{B}$,

$$
\begin{gathered}
V_{0}(t)=0, \\
V_{N+1}(t)=V_{0} \sin \omega t .
\end{gathered}
$$

The fourth-order Runge-Kutta method was used, with an integration step of $\Delta t=\pi / 500 \omega$ (1000 steps per period). We care about the energy distribution after the system reaches steady state. If higher-order energy terms are neglected, $\left\langle V_{n}^{2}\right\rangle$ is proportional to the mean energy stored in the capacitor $(\langle\rangle$ indicates a temporal average). In the harmonic approximation, the mean energy of the capacitor is equal to the sum of the mean energy of all the inductors in each cell. Therefore, we only need to consider $\left\langle V_{n}^{2}\right\rangle$. We normalized $\left\langle V_{n}^{2}\right\rangle$ by dividing by the mean square of the driving voltage, $V_{0}^{2} / 2$. This yields the dimensionless mean energy:

$$
E_{n}=2\left\langle V_{n}^{2}\right\rangle / V_{0}^{2} .
$$

The results when the driving input is at End A are shown by the solid curve in Figure 3. It can be seen that the energy flows pass the interface and enter Line b. For driving at End $\mathrm{B}$, attenuation occurs in Line $\mathrm{b}$, and Line a receives no energy as a result (see the dotted curve in Figure 3).

By comparison, it can be seen that the energy flow is asymmetric across the two directions. If we treat energy flow through the interface as conduction, the system conducts when the driving is input at End A, but insulates when driven at End $\mathrm{B}$. The entire coupled $L C$ transmission line serves as a rectifier.

Physically, when driving at End A and the $\omega$ satisfies eq. (6), the fundamental wave can propagate in Line a. However, high-order harmonic waves, mainly the second-order harmonic wave, are generated through nonlinearity. The second-order harmonic wave, at frequency $2 \omega$, satisfies eq. (7). Therefore, it can propagate in Line $b$ after passing through $L_{\text {int }}$, which results in a non-zero energy flux through the interface. When the input end is changed, $\omega$ is outside of Line b's passband, and the fundamental wave decays exponentially, which prevents energy flux through the interface. This is very similar to Liang et al.'s acoustic diode [8] in principle.

To verify the physical mechanism mentioned above, we calculated the power spectral density for the voltages of the 20th and 40th cells for driving input at End A.

From Figure 4, it can be seen that there are harmonic waves present (second and third harmonics) in $V_{20}$, and essentially the second harmonic is the only one present in $V_{40}$. At the 40th node, the fundamental wave is not present (see Figure 4(b)) because it is outside the passband of Line b.

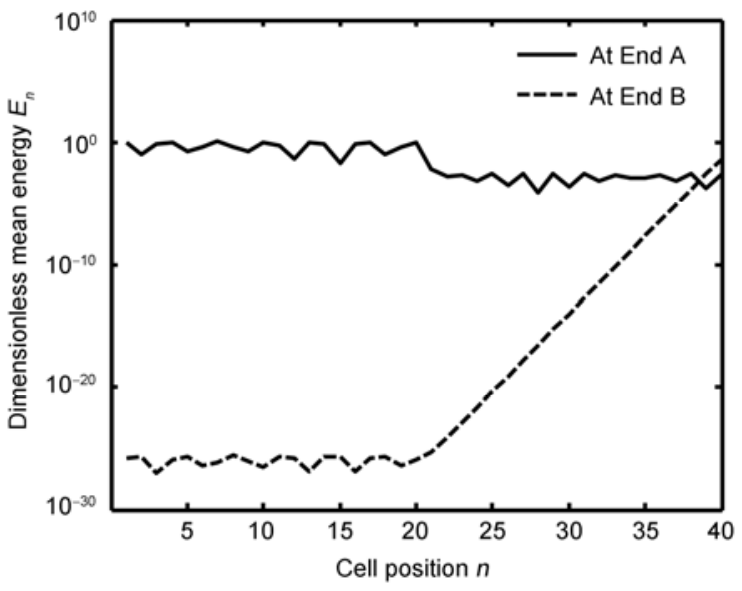

Figure 3 Energy distributions for driving signals at the two different input ends.
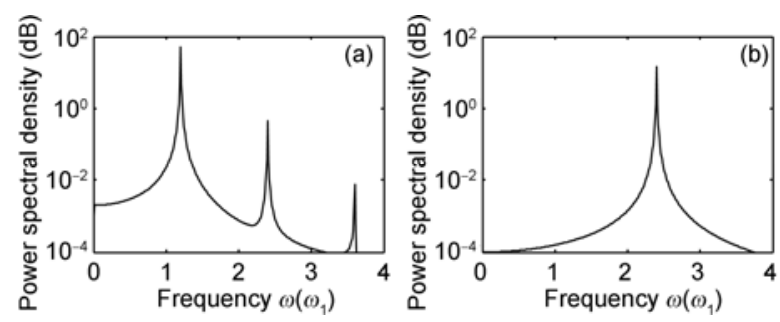

Figure 4 Power spectral density. (a) $n=20$; (b) $n=40$.

The third harmonic is also not present for the same reason. Only the second harmonic wave passes through Line b, which leads to conduction for the whole system.

The occurrence of asymmetric energy flow is dependent on the driving frequency. Furthermore, we calculated the energy distribution in the coupled line for four driving frequencies: $\omega=0.9 \omega_{1}, \omega=1.3 \omega_{1}, \omega=1.6 \omega_{1}$ and $\omega=2.2 \omega_{1}$.

The driving voltage, $\omega=0.9 \omega_{1}$, is outside the either passband for both transmission lines. Therefore, it cannot propagate in either line, and the system is an insulator in both directions. The driving frequency, $\omega=1.3 \omega_{1}$, satisfies eqs. (6) and (7), and the system behaves as a rectifier (solid curve in Figure 5). Although the waves at $\omega=1.6 \omega_{1}$ and $\omega=2.2 \omega_{1}$ can propagate in Lines a and b, respectively, neither allows for conduction across the entire system. We have marked the frequencies that satisfy eqs. (6) and (7) by the shaded region in Figure 2.

\section{Dependence of parameters}

Here, we define a variable called the mean transmission energy density, $E_{\mathrm{p}, i}(i=\mathrm{A}, \mathrm{B})$. Its value is equal to the total mean energy passing through the interface. If the system is driven at End A,

$$
E_{\mathrm{p}, \mathrm{A}}=\frac{1}{n_{\mathrm{b}}} \sum_{n \in \mathrm{b}} E_{n},
$$



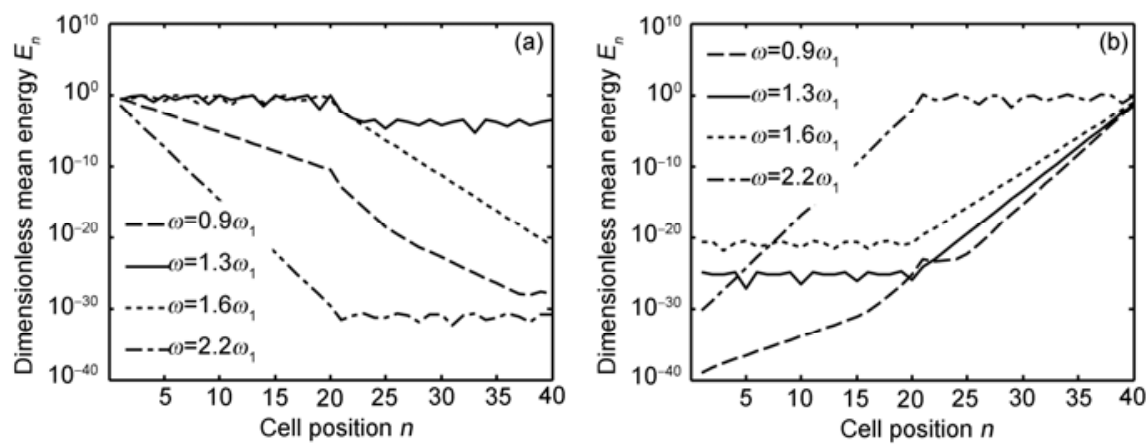

Figure 5 Energy distribution for different driving frequencies. (a) Driven at End A; (b) driven at End B.

where $n_{\mathrm{b}}$ is the total number of cells in sub-line b, and $E_{n}$ is from eq. (12). If the system is driven at End B,

$$
E_{\mathrm{p}, \mathrm{B}}=\frac{1}{n_{\mathrm{a}}} \sum_{n \in a} E_{n},
$$

with $n_{\mathrm{a}}$ being the total number of cells in sub-line a. For the conducting condition $\left(E_{\mathrm{p}, i}>0\right)$, the larger $E_{\mathrm{p}, i}$ is, the larger the energy flux is. For the insulating condition $\left(E_{\mathrm{p}, i}=0\right)$ the energy flux is zero. Using the mean transmission energy density, we investigated the dependence of the asymmetric energy flow on the parameters of the system, such as the driving amplitude and coupling strength.

\subsection{Driving amplitude}

Allowing the driving frequency to satisfy eqs. (6) and (7), we set $\omega=1.2 \omega_{1}$. The numerical calculation shows that $E_{\mathrm{p}, \mathrm{A}}$ increases with the amplitude (see Figure 6). The nonlinear effect becomes stronger as the amplitude increases, which leads to more energy being transferred to the higher-order harmonic waves from the fundamental wave. The frequency of the second harmonic wave is in the passband of Line b, which forms a finite energy flux through the coupled line. Therefore, the higher the amplitude of the second harmonic wave, the larger the energy flux is. However, $E_{\mathrm{p}, \mathrm{B}}$ remains zero.

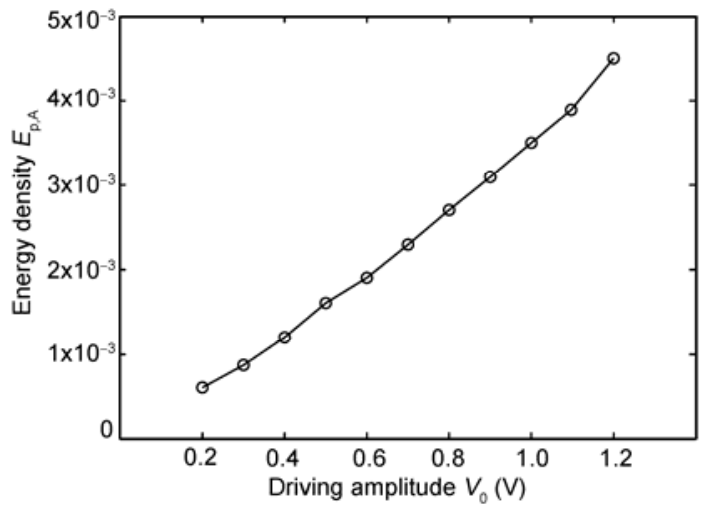

Figure $6 E_{\mathrm{p}, \mathrm{A}}$ versus $V_{0}$. Main parameters: $N=40, L_{\mathrm{int}}=4.0 L_{0}, g=1.2 \times 10^{-5}$ $\Omega^{-1}, \omega=1.2 \omega_{1}$.

\subsection{Coupling strength}

During thermal rectification, the coupling strength is critical to the asymmetric energy flow. In strong and weak conditions, the coupled Frenkel-Kontorova chains behave differently [1,9]. While $E_{\mathrm{p}, \mathrm{B}}$ keeping at zero, we calculated the dependence of $E_{\mathrm{p}, \mathrm{A}}$ on $L_{\text {int }}$ (Figure 7). As $L_{\text {int }}$ increases, $E_{\mathrm{p}, \mathrm{A}}$ initially increases, then reaches the maximal when $L_{\text {int }}=2.5 L_{0}$, and finally decreases. When $L_{\text {int }}$ is small, the coupling is strong; the two sub-lines interfere with each other's passband. In this case, our analysis, which is based on two uncoupled infinite lines, is no longer valid. However, if $L_{\text {int }}$ is too large, the coupling will be too weak, and the energy flux across the interface will be negligible. In practice, weak coupling is the most common situation.

\subsection{Cell numbers}

Here, we discuss the effect of the cell number. Our calculation shows that, $E_{\mathrm{p}, \mathrm{A}}$ decreases with the cell number, except when $N=60$ (Figure 8). It is likely that the increase of total dissipation is the main cause. We do not consider $E_{\mathrm{p}, \mathrm{B}}$ because it is nearly zero. A sufficient number of cells to accurately model the bandpass are needed. We find that the asymmetry weakens with increasing cell number, when dissipation is considered. However, we do not consider the effect of the cell number at a non-dssipation condition here.

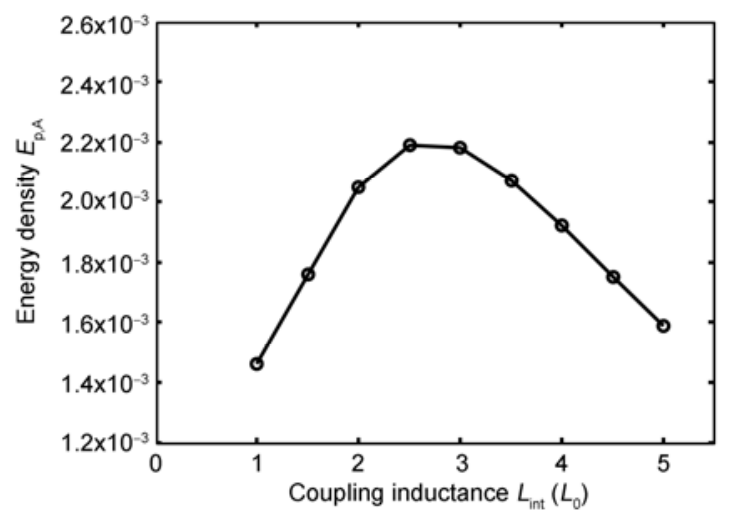

Figure $7 \quad E_{\mathrm{p}, \mathrm{A}}$ versus $L_{\mathrm{int}}$. Main parameters: $V_{0}=0.6 \mathrm{~V}$; other parameters are the same as those in Figure 6. 


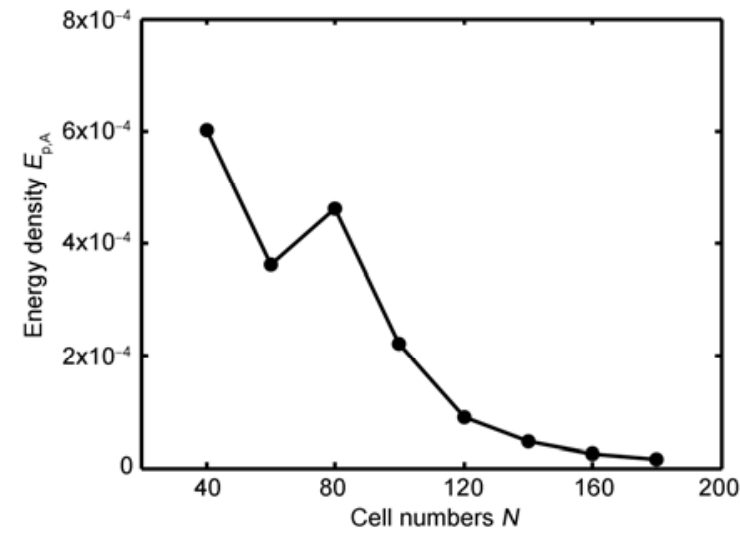

Figure $8 E_{\mathrm{p}, \mathrm{A}}$ changing with $N$. Main parameters: $V_{0}=40, L_{\mathrm{int}}=4.0 L_{0}$, $g=1.2 \times 10^{-5} \Omega^{-1}, \omega=1.2 \omega_{1}$.

\section{Discussion and conclusion}

We have studied energy flow in a coupled nonlinear $L C$ transmission line excited by a mono-frequency voltage at one end. Because of nonlinearity, high-order harmonic waves form in the first sub-line in one direction. The second-order harmonic propagates thorough the other sub-line, which leads to energy transmission. However, when the system is driven in the other direction, the fundamental wave decays exponentially in the first sub-line and no harmonic wave forms, which means that no energy transmission occurs. The energy flow is asymmetric. The overlap of the frequency and the passband of the sub-line determines the propagation or attenuation of the wave. If the driving frequency is in the shaded area shown in Figure 2, asymmetric energy flow may occur. The dependence of the system on various parameters was studied in our numerical simulation. We found that the asymmetry increases with the driving amplitude. The coupling inductance should be properly chosen to yield the greatest asymmetry. The energy flow will be attenuated if the coupling inductance is too large. However, if the coupling inductance is too small, the energy flow will also be attenuated.

For thermal flux, the cell energy would correspond to the temperature. In this case, we would connect the end of the coupled line to a heat bath. This corresponds to a complex voltage including a number of frequencies, instead of only one. As long as the frequency band of the complex voltage contains frequencies that satisfy eqs. (6) and (7) (the shaded area in Figure 2), an asymmetric energy flux will be possible. However, frequencies outside the shaded area will not form energy flows in the coupled line in either direction. Therefore, asymmetric energy flux can still be observed when a complex voltage serves as the driving voltage. We aim to provide the theoretical foundation for experimental verification, which is currently being performed.

This work was supported by the National Natural Science Foundation of China (10774072, 10974095).

1 Li B, Wang L, Casati G. Thermal diode: Rectification of heat flux. Phys Rev Lett, 2004, 93: 184301

2 Segal D, Nitzan A. Spin-Boson thermal rectifier. Phys Rev Lett, 2005, 94: 034301

3 Chang C W, Okawa D, Majumdar A, et al. Solid-state thermal rectifier. Science, 2006, 314: 1121-1124

4 Chen W Z, Zhu Y F, Lv L. Observations of impurity-soliton interactions in driven Frenkel-Kontorova chains. Phys Rev B, 2003, 67: 184301

5 Yemele D, Marquie P, Bilbault J M. Long-time dynamics of modulated waves in a nonlinear discrete LC transmission line. Phys Rev E, 2003, 68: 016605

6 Marquie P, Bilbault J M, Remoissenet M. Generation of envelope and hole solitons in an experimental transmission line. Phys Rev E, 1994, 49: 828-835

7 Marquie P, Bilbault J M. Bistability and nonlinear standing waves in an experimental transmission line. Phys Lett A, 1993, 174: 250-254

8 Liang B, Yuan B, Cheng J C. Acoustic diode: Rectification of acoustic energy flux in one-dimensional systems. Phys Rev Lett, 2009, 103: 104301

9 Hu B, Yang L, Zhang Y. Asymmetric heat conduction in nonlinear lattices. Phys Rev Lett, 2006, 97: 124302

Open Access This article is distributed under the terms of the Creative Commons Attribution License which permits any use, distribution, and reproduction in any medium, provided the original author(s) and source are credited. 